\title{
Analogue optical links experiences in the framework of the SKA/BEST activities *
}

\begin{abstract}
F. Perini
INAF-Istituto di Radioastronomia, Via Fiorentina 3508/B, Medicina (BO), Italy email: f.perini@ira.inaf.it

Abstract. In the framework of the SKADS/BEST activities the analogue optical link solution in the signal transportation from the antennas to the back end has been investigated by IRA-INAF. Many aspects have been faced as the design and the characterization of both commercial and ad hoc conceived devices. Fibre cable and connectors have been tested as well. The BEST demonstrator has been using to test such a technology on the field. It is a natural bridge towards SKA especially to the signals transportation in the low frequency bands and systems (AA-lo and AA-high).
\end{abstract}

\section{Introduction}

The BEST demonstrator is based on the re-instrumentation of several antennas of the Northern Cross radio telescope. The distance between the front ends, installed on the antennas focal lines, and the receiver room, inside the main building of the Medicina station, could reach several hundred meters. Different signal transportation systems could be adopted with different technologies and/or system approaches (i.e. coaxial or optical cable, analogue or digital link). Cost and reliability prediction analysis of the whole receiver chain indicated an analogue optical link, which brings directly the RF signal from the antennas to the central processing room, as the best solution (Catelani et al. 2009). For that reason we investigated and experienced both the optical transmission medium (both optical cables and optical connectors) and the electro-optic devices suitable for the application on the BEST demonstrator.

\section{The optical cables}

The use of monomode fibres for analogue optical transmission is well know and established. Respect to multimode fibres, they allow broader bandwidth and/or longer distance. Also they present a better phase stability versus bending, which is important for installation on steerable antennas. In order to better understand the right optical cable to adopt, a measurement system was set up. In particular, we compared the thermal coefficient of delay (TCD, see Eq. 1) of two optical trunks, both $200 \mathrm{~m}$ long, putting them inside a temperature chamber. The two cables were a tight one and a loose one. The results are plotted in Fig. 1. As can be noticed the loose buffered fibres are more stable than the tight ones. Moreover, since in an array application the relative stability among the receiver chains is important too, loose fibres are preferable due to their better uniformity.

$$
T C D=\frac{10^{6}}{t_{\text {nom }}} \frac{\Delta t}{\Delta T}=\frac{10^{6}}{\phi_{\text {nom }}} \frac{\Delta \phi}{\Delta T}
$$

^ This work was supported by the European Commission Framework Program 6, Project SKADS, Square Kilometre Array Design Studies (SKADS), contract no 011938.

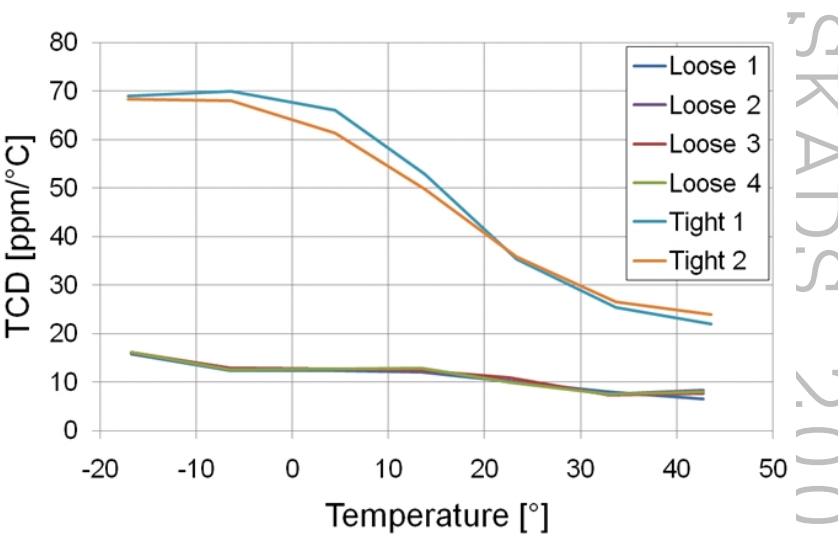

Fig. 1: Measured TCD for Loose and Tight optical cables

\section{Optical connectors}

In analogue optical links where both noise and dynamic range performances are important, it is imperative to avoid any light back reflection into the laser source. For that reason, to adopt optical connectors which present high return loss, like the APC (Angle Polished Connector) ones, which have a minimum RL of $70 \mathrm{~dB}$, is mandatory. For the large use of the connectors in an array link application, in order to reduce costs, without losing in performance, the right optical connector must be chosen. After a market research we choose LSA(DIN)/APC connector by Diamond, for the application on the antenna, due to its optimal performance in terms of mechanical stability. For the receiver side, we choose standard SC/APC connectors. For an indoor application, without any moving part, they are the cheapest solution (6 euro) which allows, at the same time, the maximum integration. Due to the high cost of the LSA(DIN) connectors (about 40 euro), a migration to the FC/APC standard is under evaluation: they have similar cost to the SC/APC one, but with good performance in terms of mechanical stability. 


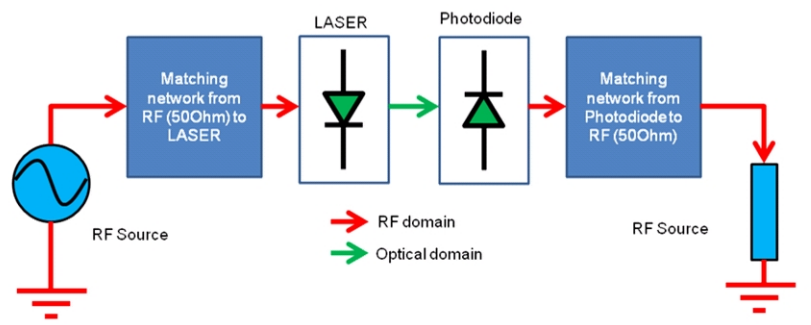

Fig. 2: IMDD link

\section{Electro optical devices: IRA optical link}

In order to satisfy both link performances and link cost, a research on the appropriate link architecture (direct modulation or external modulation of the laser source) has been done. For low frequency applications (even several GHz), the intensity modulation-direct detection (IM-DD) has resulted to be the best compromise (Fig. 2). Once selected the architecture, a link model was studied in order to better understand the reachable link performances and, at the same time, to guide us in the right devices selection (laser and photodiode), see Eq.2 and Eq.3:

$$
\begin{gathered}
G_{a}[d B]=10 \log \left[\frac{g_{\text {opt }}^{2}\left(k_{1} k_{1}\right)^{2}}{4} \frac{R_{\text {out }}}{R_{\text {in }}}\right] \\
N F[d B]=10 \log \left[2+\frac{4 R_{\text {in }}}{g_{\text {opt }}^{2}\left(k_{1} k_{1}\right)^{2} R_{\text {out }}}+\right. \\
\left.+\frac{R_{\text {in }}\left[I_{D}^{2} R I N+2 e\left(I_{D}+I_{\text {dark }}\right)\right]}{k T g_{\text {opt }}^{2}\left(k_{1}\right)^{2}}\right]
\end{gathered}
$$

were $k_{1}$ and $k_{2}$ are the input and output matching losses, $R_{\text {in }}$ and $R_{\text {out }}$, respectively, the equivalent input resistance and the equivalent output resistance and $g_{o p t}^{2}=\eta_{\text {Laser }} \mathfrak{R}_{\text {PIN }} / A_{\text {opt }}$ is, with obvious meaning of the symbols, the optical gain. The selected devices are both from Sumitomo. The main characteristics are reported below:

- Laser DFB 1310 nm MQW SPV4260

- $\mathrm{RIN}=-155 \mathrm{~dB} / \mathrm{Hz}$

- $\eta=0.2 \mathrm{~mW} / \mathrm{mAz}$

- $\mathrm{I}_{\mathrm{th}}=8 \mathrm{~mA}$

- PIN Photodiode SPV3313

- $\mathfrak{R}=0.9 \mathrm{~mA} / \mathrm{mW}$

- $\mathrm{I}_{\mathrm{dark}}=1 \mathrm{nA}$

- $\mathrm{F}_{\max }=2 \mathrm{GHz}$

Regarding the optical TX design, a simple resistive matching between the RF input $(50 \Omega)$ and the laser, which present a low equivalent input impedance (typically $5 \div 10 \Omega$ ), has been adopted. Moreover the transmitter includes an automatic power control circuits, which keeps constant the optical power emitted by the optical source. Since lasers are susceptible to sudden switch-on and switch-off operations, the circuit allow also slow on/off-switching of the optical sources when the bias is applied (or removed) to the transmitter (see Fig. 3). For the optical receiver, a simple passive scheme has been adopted. The

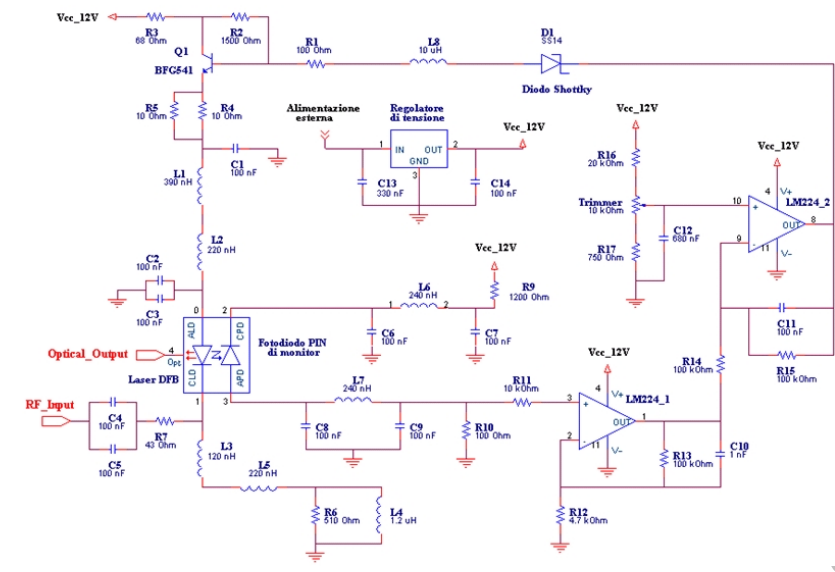

Fig. 3: IRA Optical TX circuit schematic

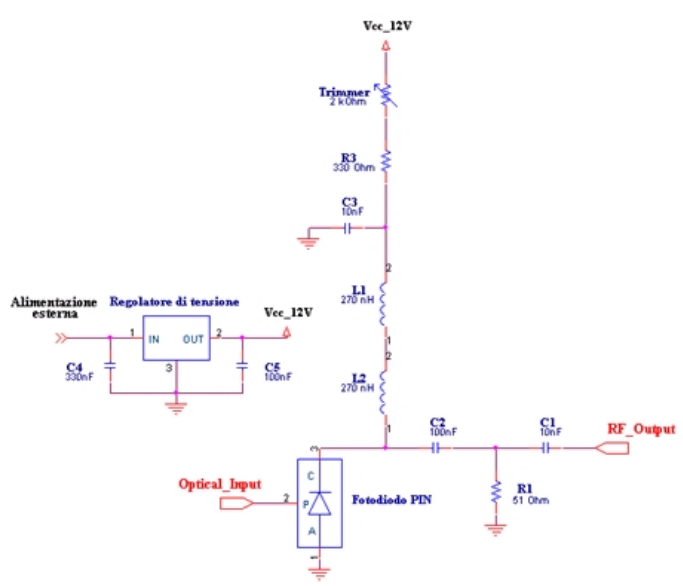

Fig. 4: IRA Optical RX circuit schematic

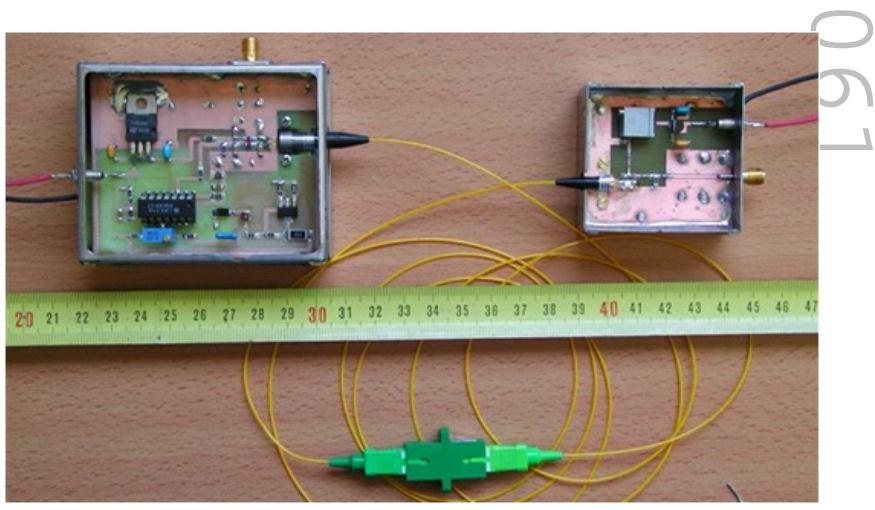

Fig. 5: IRA optical link prototype

RF signal, once recovered in the electrical domain by the photodiode, is not amplified by a trans-impedance amplifier, but feed directly into a passive matching network to the $50 \Omega$ (see Fig. 4). The prototype has been mounted (Fig.5) and measured (see Fig. 6 and Table 1) in the Medicina labs. Even if designed and optimised for the BEST-2 band (16 MHz @ $408 \mathrm{MHz}$ ), the IRA optical link has shown good performance also in the wider 

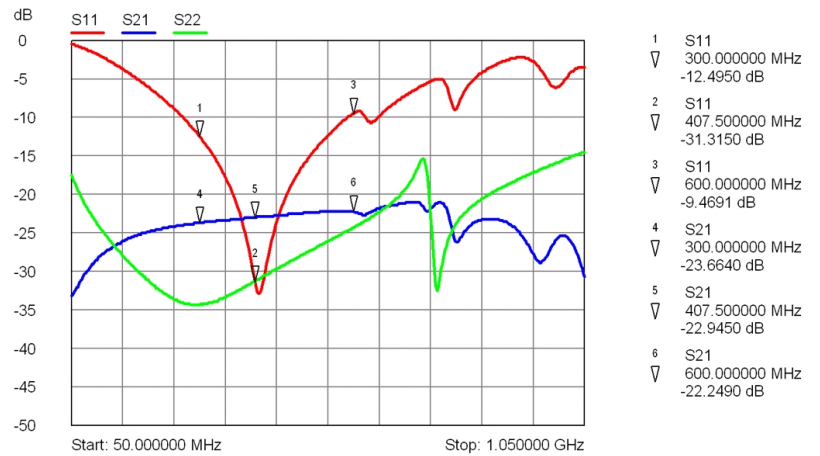

Fig. 6: IRA optical link S-parameters measurements

Table 1: IRA optical link measurement summary

\begin{tabular}{|c|c|c|c|}
\hline \multicolumn{2}{|c|}{$\begin{array}{c}\text { Link optimised at } \\
16 \mathrm{MHz} @ 408 \mathrm{MHz}\end{array}$} & \multicolumn{2}{|c|}{$\begin{array}{l}\text { Link (not optimised) in } \\
(300-600) \mathrm{MHz} \text { band }\end{array}$} \\
\hline Gain $[\mathrm{dB}]$ & -22.9 & Gain $_{\min }[\mathrm{dB}]$ & -23.7 \\
\hline Gain flatness [dB] & $<0.1$ & Gain $_{\max }[\mathrm{dB}]$ & -22.1 \\
\hline $\begin{array}{l}\text { Input Return } \\
\text { Loss }[\mathrm{dB}]\end{array}$ & $>28.6$ & $\begin{array}{l}\text { Input Return } \\
\text { Loss }[\mathrm{dB}]\end{array}$ & $>9.5$ \\
\hline $\begin{array}{l}\text { Output Return } \\
\text { Loss }[\mathrm{dB}]\end{array}$ & $>30.9$ & $\begin{array}{l}\text { Output Return } \\
\text { Loss }[\mathrm{dB}]\end{array}$ & $\begin{array}{l}>24.9 \\
>24.9\end{array}$ \\
\hline Input IIP3 [dBm] & +32.8 & Input IP3 [dBm] & $>+30$ \\
\hline Noise figure $[\mathrm{dB}]$ & 31 & Noise figure $[\mathrm{dB}]$ & $<+31.3$ \\
\hline
\end{tabular}

(300 - 600) MHz band. The estimated link cost was 500 euro for a 400 pieces production. A reliable production should be investigated and, up to now, represents the main risk factor for an "all IRA" manufacturing.

\section{The collaboration with the industry}

\subsection{The collaboration with Andrew Wireless Systems}

To reduce the costs of the TX, mainly dominated by the laser cost, opening a direct link with factories (for example Sumitomo) that produce laser sources, overcoming local sellers and representatives, would be necessary. With the low volume of BEST, it was not possible to do that. Moreover, to test the produced devices, an optical lab would be necessary. The instrumentations are very expensive and only a massive production for SKA, or other SKA large pathfinder, could justify such effort. For these reason we decided to customise a commercial link by Andrew Wireless System, which has been adopted for the various BEST systems. The Andrew's link is optimised for working in the band $(100-1000) \mathrm{MHz}$ (see both Fig. 7 and Table 2). The costs is about 700 euro, for the TX, and 180 euro, for the RX.

During some astronomical observation made with BEST-1, equipped with 4 Andrew's optical link, a ripple on the detected traces of some sources has been noticed (see the reed circle in Fig.8). Thanks to a measurement system (see Fig. 9) able to reproduce slow environmental temperature variations, similar

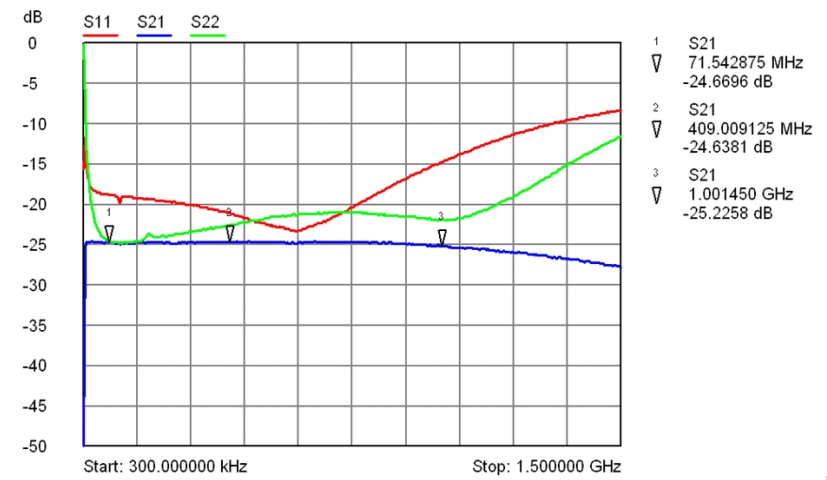

Fig. 7: ANDREW optical link S-parameters

Table 2: ANDREW optical link summary

\begin{tabular}{|l|c|}
\hline \hline \multicolumn{2}{|c|}{$\begin{array}{c}\text { TFTY2324w+TFRY501 } \\
100-1000 \mathrm{MHz} \text { band }\end{array}$} \\
\hline Gain [dB] & -25 \\
\hline Gain flatness [dB] & \pm 0.75 \\
\hline Input Return Loss [dB] & $>15$ \\
\hline Output Return Loss [dB] & $>20$ \\
\hline Input IIP3 [dBm] & +33 \\
\hline Noise figure [dB] & 36 \\
\hline
\end{tabular}

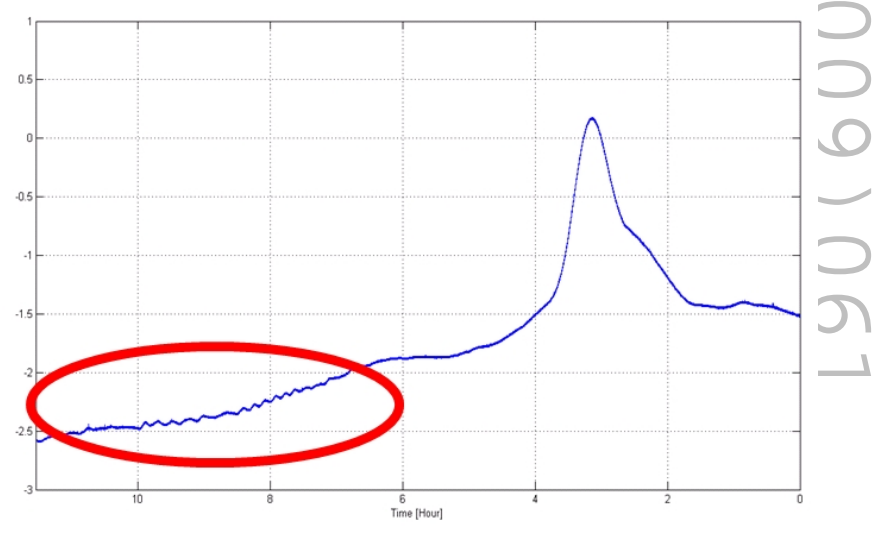

Fig. 8: Ripple of a detected transit of Cas-A with a single BEST-1 RX

to the one in which the electronics mounted on the antennas is normally operating, the Andrew'optical TX was found susceptible to ripple generation, both on the link insertion gain and phase (Perini \& Ghelfi 2008). We estimated a ripple amplitude of about $\pm 0.02 \mathrm{~dB}$, with a variable period in the range from 10 to 15 minutes. In order to understand the possible effect of such ripple on the BEST performances, several astronomical tests have been done. First preliminary results (see Fig. 10) seem indicate that the instrument, when operates as interferometer, is not influenced. The ripple effect on a beamformer is still to investigate. 


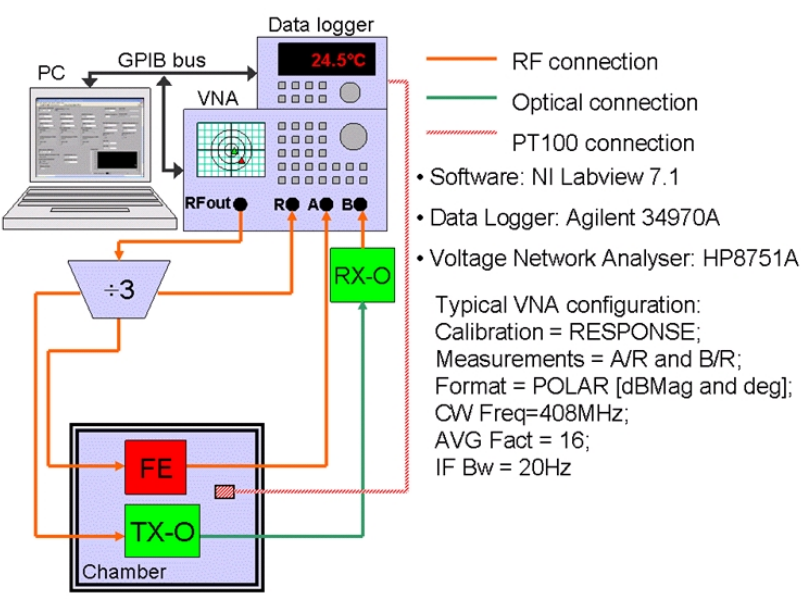

Fig. 9: Ripple measurement set up

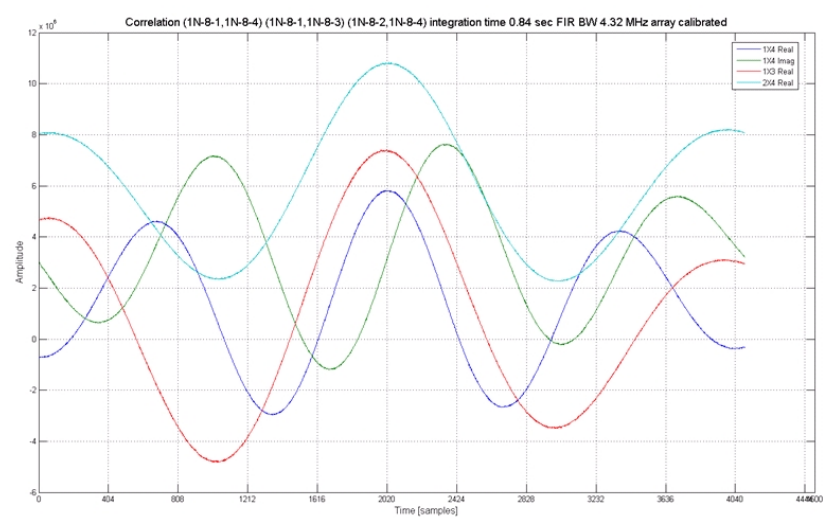

Fig. 10: BEST complex fringes of Cas-A source. No ripple effects are detectable in more than one hour of data acquisition

\subsection{New opportunities}

Since the Andrew's local factory has been moved to another country, a research of new local factories of analogue optical links is underway. After some contacts, a customised link by Tekmedia factory has been produced and tested. The TX and RX pair cost about 700 euro. Preliminary measurements are interesting (see Fig.11). The bandwidth is more than $2 \mathrm{GHz}$ ( $\mathrm{RX}$ post amplified) and the noise is very good below $1 \mathrm{GHz}$ (i.e. $28 \mathrm{~dB} @ 408 \mathrm{MHz}$ ). Moreover the Tekmedia TX is ripple free (see Fig.12).

\section{Conclusions}

A valuable experience in the design and characterisation of analogue optical links has been gained in the frame of SKADS activities. Moreover, the link application on the BEST demonstrator has represented a useful and unique opportunity to test such technology on the field with real astronomical observations.

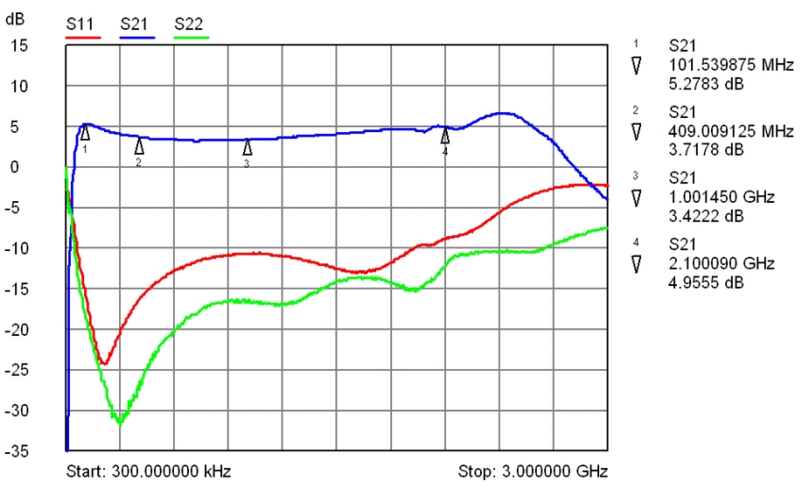

Fig. 11: Tekmedia measured S-parameter

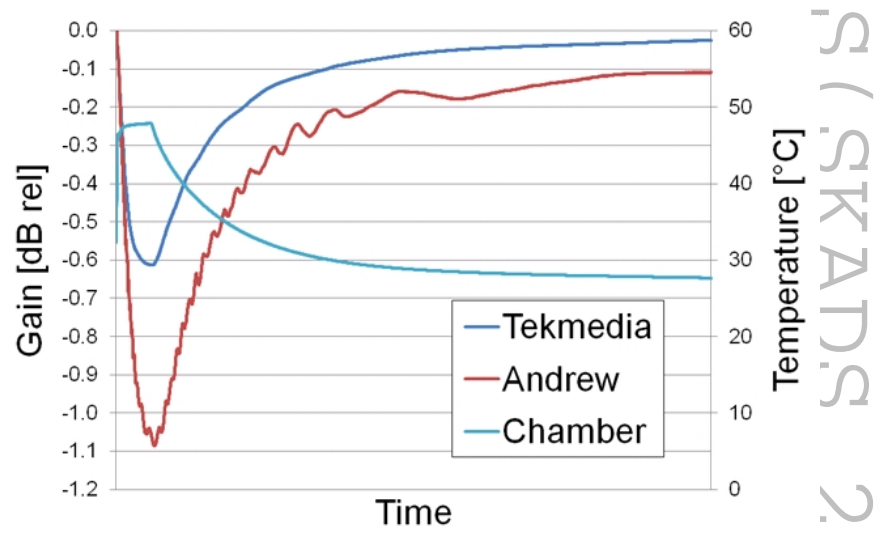

Fig. 12: Ripple measurement

\section{References}

M.Catelani, V.L. Scarano, R. Singuaroli, S. Montebugnoli, G. Bianchi, F. Perini, I. Trotta, Northern Cross Radiotelescope: test and measurements of reliability performance on radioreceiver chains, IEEE Transaction on instrumentation and measurement, vol.58, No. 10, October 2009

F. Perini, F. Ghelfi, A methodology to reproduce slow operative temperature transitions and to measure their induced effects on receivers for radio astronomical applications. Proceedings of the $16^{\text {th }}$ IMEKO TC4 Symposium $\& 13^{\text {th }}$ Workshop on ADC Modelling and Testing, Florence, 22-24 September 2008 